\title{
Monotone Percolation and The Topology Control of Wireless Networks
}

\author{
Anxiao (Andrew) Jiang \\ Electrical Engineering Department \\ California Institute of Technology \\ Pasadena, CA 91125, U.S.A. \\ Email: jax@paradise.caltech.edu
}

\author{
Jehoshua Bruck \\ Electrical Engineering Department \\ California Institute of Technology \\ Pasadena, CA 91125, U.S.A. \\ Email: bruck@paradise.caltech.edu
}

\begin{abstract}
This paper addresses the topology control problem for large wireless networks that are modelled by an infinite point process on a two-dimensional plane. Topology control is the process of determining the edges in the network by adjusting the transmission radii of the nodes. Topology control algorithms should be based on local decisions, be adaptive to changes, guarantee full connectivity and support efficient routing. We present a family of topology control algorithms that, respectively, achieve some or all of these requirements efficiently. The key idea in our algorithms is a concept that we call monotone percolation. In classical percolation theory, we are interested in the emergence of an infinitely large connected component. In contrast, in monotone percolation we are interested in the existence of a relatively short path that makes monotonic progress between any pair of source and destination nodes. Our key contribution is that we demonstrate how local decisions on the transmission radii can lead to monotone percolation and in turn to efficient topology control algorithms.
\end{abstract}

Keywords: Combinatorics, Graph theory, Probability, Topology, Topology Control, Wireless network.

\section{INTRODUCTION}

The topology of a wireless network is the basis for its performance. Nearly all the important properties - connectivity, data-transmission efficiency, etc. - rely on it. In this paper, we consider large wireless networks that are modelled by an infinite point process on a two-dimensional plane. We use the following simplified and commonly used topology model for wireless networks [1], [8], [12], [16], [22]: the coverage area of a node is a disk; if the coverage radius of a node $u$ is $r$, then $u$ has an outgoing edge to each node whose Euclidean distance to $u$ is less than or equal to $r$. (See Fig. 1 for examples.) The topology control problem of a wireless network is for the nodes to select their coverage radii, which will determine the network topology.

It is very desirable that the topology control problem can be solved with a localized construction, meaning that every node can decide on its coverage radius by using only the information about its nearby neighbors. This is not only for reducing the complexity of the topology control process, including the complexity in computational time, space and the exchanged information flows among nodes, but also because with a localized construction, when changes happen (e.g., when one (a)

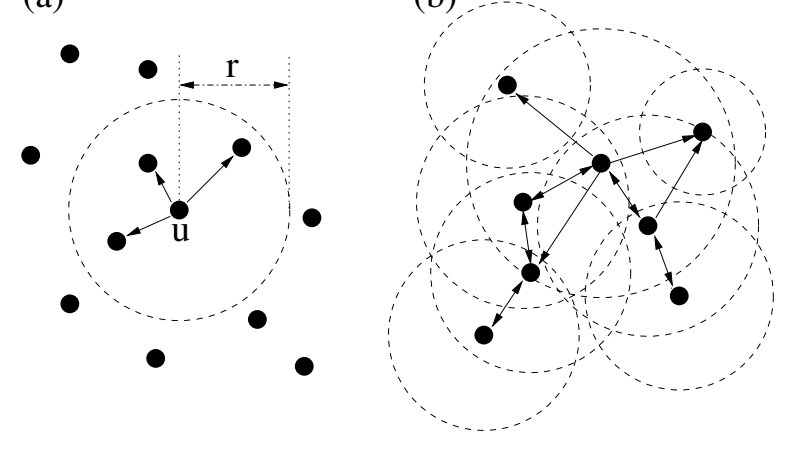

Fig. 1. (a) The wireless node $u$ has coverage radius $r$. $u$ has an outgoing edge to every node in the disk of radius $r$. (b) The wireless nodes cover areas (disks) as shown, which result in the directed edges.

or more nodes move away), nodes can easily update their coverage radii.

Despite the requirement on using localized topology control methods, it is desirable at the same time that the network topology has good global properties. Routing, one of the key network functions, can serve as an example here. For a large-scale wireless (and possibly mobile) network, the control messages for forming and maintaining routing tables can easily consume a large portion of the network capacity. To make routing scalable to the network size, geographical routing or compact routing schemes similar to it are often used [3], [13], [14], [15], [18]. In geographical routing, when a node is forwarding a message, it selects a neighbor whose distance to the destination is closer than its own as the next hop in the routing path. If a node forwarding a message has no neighbor closer to the destination, then we call it a dead-end. To facilitate geographical routing, the network is desired not only to be strongly connected, but to contain no dead-ends as well - both of which are global properties. Traditionally, to deal with dead-ends, path-recovery mechanisms such as the perimeter routing mechanisms based on planar subgraphs [1], [3], [7], [13] — are used. Such mechanisms ensure correct delivery, but have the cost of extra routing length, under-utilization of certain communication links and possibly unbalanced load. Constructing network topologies 
suitable for geographical routing is a very valuable topic. An ideal topology will be one that eliminates dead-ends for all destinations.

It is a challenging task to construct wireless networks with good global topological properties by using only localized constructions. The objectives that a good topology control algorithm should try to achieve include:

- Localized construction: every node determines its coverage radius by using only the information about the nearby nodes.

- Strong connectivity: a network is strongly connected if there is a directed path from any node to any other node.

- Small node degree: the degree of a node $v$ is defined to be the number of nodes (except $v$ itself) within the coverage radius of $v$ - namely, the number of outgoing edges that $v$ has. If $v$ has a small degree, then it means that when $v$ transmits a message, its signal interferes with only a small number of neighbors.

- Small coverage radius: it leads to small power consumption.

- Small hop distortion: for any two nodes $u$ and $v$, we use $h(u, v)$ to denote the minimum number of hops (edges) in a directed path from $u$ to $v$, and use $d(u, v)$ to denote the Euclidean distance between $u$ and $v$. Then we define the hop distortion for the ordered node pair $(u, v)$ as $\frac{h(u, v)}{d(u, v)}$. Hop distortion measures how much the shortest path in terms of hops between a pair of nodes is stretched when it is compared to the Euclidean distance between those two nodes. We emphasize here that in this paper, we consider the hop distortion for an ordered node pair $(u, v)$ only if $h(u, v) \geq 2$. That is because if there is an edge directly from $u$ to $v$, then the hop distortion is $\frac{1}{d(u, v)}$, which approaches $\infty$ if $d(u, v) \rightarrow 0$; however it is neither necessary nor possible to make the hop distortion be smaller than $\frac{1}{d(u, v)}$ by using any alternative topology control algorithm.

- Small length distortion: for any two nodes $u$ and $v$, we use $l(u, v)$ to denote the minimum Euclidean length of a directed path from $u$ to $v$. We define the length distortion for the ordered node pair $(u, v)$ as $\frac{l(u, v)}{d(u, v)}$. Length distortion measures how much the shortest path in terms of length between a pair of nodes is stretched when it is compared to the Euclidean distance between them.

An example of hop distortion and length distortion is shown in Fig. 2.

- No dead-ends for geographical routing: such a property greatly facilitates geographical routing.

The above objectives comprehensively describe the connectivity properties of a wireless network. Traditional topology control methods often achieve only a few of them. For example, with the common practice of making all the nodes have the same coverage radius, the network becomes a unitdisk graph. For an infinitely large network where the node positions follow a random point process, such as a Poisson

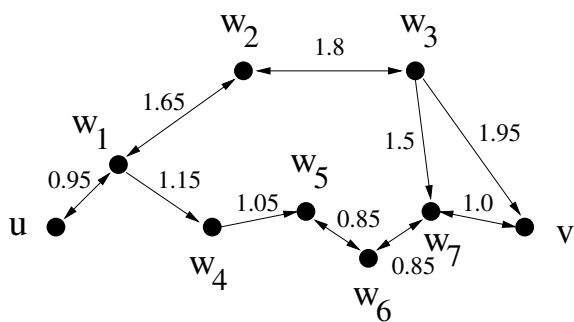

Fig. 2. In the above network, the number beside each edge is the Euclidean distance between the two endpoints of that edge. The Euclidean distance between $u$ and $v$ is 4.85. Then, the shortest path from $u$ to $v$ in terms of hops is $u \rightarrow w_{1} \rightarrow w_{2} \rightarrow w_{3} \rightarrow v$, which has 4 hops. The shortest path from $u$ to $v$ in terms of length is $u \rightarrow w_{1} \rightarrow w_{4} \rightarrow w_{5} \rightarrow w_{6} \rightarrow w_{7} \rightarrow v$, which has length $0.95+1.15+1.05+0.85+0.85+1.0=5.85$. So for the ordered node pair $(u, v)$, the hop distortion is $\frac{4}{4.85}=0.82$, and the length distortion is $\frac{5.85}{4.85}=1.21$.

point process, a unit-disk graph essentially cannot be strongly connected, has very large hop distortions and length distortions for certain node pairs, and has infinitely many dead-ends. In this paper, we present a family of topology control algorithms that, respectively, achieve some or all of the above objectives efficiently. To the best of our knowledge, this is the first time algorithms are presented that achieve this broad set of objectives.

We call our algorithms Monotone Percolation Algorithms, because they share the following common features: between any pair of source and destination nodes, there exists a relatively short path that makes monotonic progress; every node can reach infinitely many other nodes (i.e, the percolation property) through directed paths. Such a percolation model bears both clear similarity and distinction when compared to classic percolation processes [6], [10], [19]; in particular, it should be contrasted with the oriented percolation studied previously [10] [19].

Our key contribution is that we demonstrate how local decisions on the transmission radii can lead to monotone percolation and in turn to efficient topology control constructions. Our algorithms use three basic tools.

The first tool is called cone-angle [16], [22], defined as follows. Say a node $u$ has $m$ out-going edges, respectively to $v_{1}, v_{2}, \cdots, v_{m}$. We assume $v_{1}, v_{2}, \cdots, v_{m}$ have clockwise positions around $u$, as illustrated in the example of Fig. 3 (where $m=6$ ). Then the angles $\angle v_{1} u v_{2}, \angle v_{2} u v_{3}, \cdots, \angle v_{m} u v_{1}$ are called $u$ 's cone-angles. (Clearly $\angle v_{1} u v_{2}+\angle v_{2} u v_{3}+\cdots+$ $\angle v_{m} u v_{1}=2 \pi$.) In our algorithms, every node makes its coverage radius to be large enough so that its cone-angles are all smaller than a certain threshold.

The second tool is creating bi-directional edges between nodes. It means that when there is a directed edge from node $u$ to node $v, v$ increases its coverage radius large enough so that there is also a directed edge from $v$ to $u$.

The third tool is setting a lower bound on the coverage radii, namely, to make all nodes' coverage radii to be no less than a certain threshold.

In this paper, we take different combinations of the above tools and adjust their parameters, and observe how the prop- 


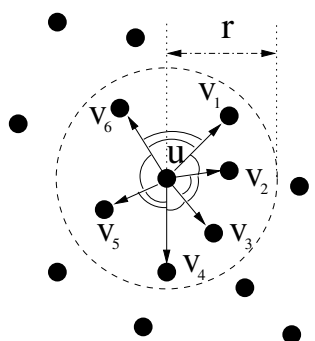

Fig. 3. The wireless node $u$ has coverage radius $r$. So $u$ has 6 outgoing edges to the 6 neighboring nodes within distance $r$.

erties of the wireless-network topology will evolve as a result.

The rest of the paper is organized as follows. In Section II, we review previous topology-control algorithms, and reveal their relationship to and difference from our algorithms. From Section III to Section VI, we introduce four correlated monotone-percolation topology control algorithms, and analyze their performance. In Section VII we present the concluding remarks.

\section{RELATED WORK}

A lot of topology-control algorithms have been proposed, and the study on their performance has been extensive [5], [8], [11], [12], [16], [17], [20], [21], [22], [23]. Those algorithms can be approximately classified into two families.

The first family of algorithms is not adaptive to the spatial variance of node densities. For example, nodes may be required to have the same coverage radius [8], or to connect to the same number of neighbors [5], [11], [23]. How to tune the parameters (e.g., coverage radius, node degree) to create an infinitely large network component or even better connectivity properties is often studied. With such algorithms, in order to get a strongly-connected network, the coverage radii or the node degrees need to approach infinity if the network size approaches infinity, which implies high power requirement and heavy signal interference, and large hop/length distortions and dead-ends often exist for geographical routing.

The second family of algorithms, in comparison, is adaptive. Nodes typically choose to have small coverage radii in areas where the node density is high, and to have large coverage radii elsewhere. Such algorithms include [12], [16], [17], [20], [21], [22]. These algorithms create better network topologies than the non-adaptive ones do. However, none of them has satisfied all the performance criteria we have proposed in Section I.

Our algorithms belong to the family of adaptive algorithms. In particular, they are close to those algorithms that use coneangles. The concept of cone-angle has been used implicitly in quite a few early topology-control works [12], [17], [21]. For example, the NFP (Nearest with Forward Progress) algorithm presented in [12] essentially chooses $\pi$ as a threshold for coneangles. In 2001, Wattenhofer, Li et al. explicitly established the concept of cone-angle in their ingenious papers [22] and [16], where they also proposed cone-based topology-control algorithms for maintaining connectivity and minimizing power consumption.

Our work differs from previous results in the usage of topology control methods and their analysis. We take flexible combinations of the topology control tools, and the main objective of this paper is to study how the network's properties change when the parameters in the tools are altered. Also our algorithms create static topologies, unlike the previous works [12], [17], [22] where the transmission radius change when a node transmits to neighbors at different distances. The networks we study are mainly directed graphs. In principle, the two-way messages between adjacent nodes for control functions can use an additional channel of relatively small bandwidth. Also, the study of networks that allow directed links shows us the limit of the performance achievable by networks.

\section{Topology CONTROL Algorithm I}

\section{A. Definition of Algorithm I}

Our first topology control algorithm, Algorithm I, is defined as follows.

Definition 1: Every node chooses its coverage radius to be the minimum value, subject to the constraint that its coneangles are all smaller than $\pi$. This topology control method is denoted by Algorithm I.

In this algorithm, and also in later algorithms, we assume that the nodes know their positions. This requirement can be met by using positioning systems such as GPS, or by using localization algorithms of good precision [4], [9].

An example of Algorithm I is shown in Fig. 4.

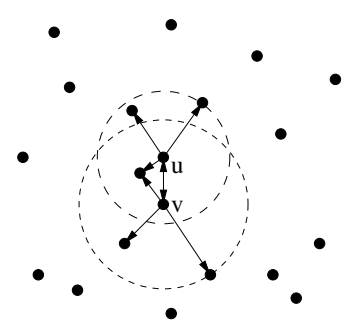

Fig. 4. This example shows how two nodes $u$ and $v$ select their coverage radii by using Algorithm $\mathrm{I}$. The radii of the two dashed circles are the coverage radii of $u$ and $v$, respectively. Neither $u$ nor $v$ can choose a smaller coverage radius, because otherwise its cone-angles would not all be smaller than $\pi$.

\section{B. Node Degree Analysis}

In this subsection we analyze the node degrees for Algorithm I. We assume the nodes are deployed on the plane with a Poisson point process. The following theorem shows the distribution of a node's degree.

Theorem 1: Assume that nodes are deployed on the plane following a Poisson point process. Let $Z$ denote the degree 
of a node in the network constructed using Algorithm I. Then for $n=1,2,3, \cdots$,

$$
\operatorname{Pr}\{Z \leq n\}=1-\frac{n}{2^{n-1}}
$$

Equivalently, $\operatorname{Pr}\{Z=1\}=\operatorname{Pr}\{Z=2\}=0$, and

$$
\operatorname{Pr}\{Z=n\}=\frac{n-2}{2^{n-1}}
$$

for $n=3,4,5, \cdots$.

Proof: We use $Z$ to denote the degree of a generic node $u$, and use $v_{1}, v_{2}, \cdots, v_{n}$ to denote the nearest $n$ nodes from $u$, where $d\left(u, v_{1}\right) \leq d\left(u, v_{2}\right) \leq \cdots \leq d\left(u, v_{n}\right)$. Without loss of generality, we let the ray starting at $u$ and going through $v_{1}$ be the positive $x$-axis, with $u$ as the origin. Let $\theta_{i}$ denote the absolute angle of $v_{i}$, that is, the counterclockwise angle from the positive $x$-axis to the ray starting at $u$ and going through $v_{i}$. It is simple to see that for $i=2,3, \cdots, n, \theta_{i}$ is an i.i.d. variable uniformly distributed in the range $[0,2 \pi)$.

The $n$ rays starting at $u$ and going respectively through $v_{1}$, $v_{2}, \cdots, v_{n}$ cut the plane into $n$ cones. $Z \leq n$ if and only if all the cone angles are smaller than $\pi$, which is equivalent to the following event: there exist $i$ and $j$ where $i \neq 1, j \neq 1$ and $i \neq j$, such that $0<\theta_{i}<\pi, \pi<\theta_{j}<\theta_{i}+\pi$, and for any $k$ where $k \notin\{1, i, j\}$, either $0<\theta_{k}<\theta_{i}$ or $\theta_{j}<\theta_{k}<2 \pi$. (We do not consider incidents of zero probability, such as having two nodes of the same absolute angle.) There are $(n-1)(n-2)$ ways to select the two indices $i$ and $j$. The probability that ' $0<\theta_{k}<\theta_{i}$ or $\theta_{j}<\theta_{k}<2 \pi$ ' equals $\left(2 \pi+\theta_{i}-\theta_{j}\right) / 2 \pi$. Therefore, when $n \geq 3, \operatorname{Pr}\{Z \leq n\}=\left(\frac{1}{2 \pi}\right)^{2} \int_{0}^{\pi} \int_{\pi}^{\theta_{i}+\pi}(n-$ 1) $(n-2)\left[\left(2 \pi+\theta_{i}-\theta_{j}\right) / 2 \pi\right]^{n-3} d \theta_{j} d \theta_{i}=1-\frac{n}{2^{n-1}}$. The rest of the proof is straightforward.

We are interested in how large the node degree is in the average case, since it is an important measure of the network's global performance. The following theorem shows that for Algorithm I, both the expectation and the variance of the node degree are small numbers. Those two values can be computed by using the degree distribution derived in Theorem 1. However we present here an alternative solution for the following reasons: the solution is intriguing and makes the degree's expectation and variance significantly easier to compute; it provides a more 'combinatorial' analysis that can be used for many generalized forms of Algorithm I.

Theorem 2: Assume that nodes are deployed on the plane following a Poisson point process. Let $Z$ denote the degree of a node in a network constructed using Algorithm I. Then $E(Z)=5, \operatorname{Var}(Z)=4$.

Proof: Let the degree $Z$, the node $u$, the nodes $v_{i}$, the number $n$ and the absolute angles $\theta_{i}$ be defined in the same way as in the proof for Theorem 1 . For $2 \leq i \leq n$, we define the number $C_{i}$ as $C_{i}=\frac{\theta_{i}}{2 \pi}$. We write $C_{i}$ in the binary form: $C_{i}=\left(0 . c_{i 1} c_{i 2} c_{i 3} \cdots\right)_{2}$. For example, if $C_{i}=0.75$, then its binary form is $C_{i}=\left(0 . c_{i 1} c_{i 2} c_{i 3} \cdots\right)_{2}=(0.110 \cdots)_{2}$. Let $\sigma$ be a permutation of the numbers in $\{2,3, \cdots, n\}$ that satisfies this property: if $\left(0.0 c_{i 2} c_{i 3} \cdots\right)_{2}<\left(0.0 c_{j 2} c_{j 3} \cdots\right)_{2}$, then $\sigma(i)<$ $\sigma(j)$. For example, if $n=4$ and $C_{2}=(0.010 \cdots)_{2}, C_{3}=$ $(0.111 \cdots)_{2}, C_{4}=(0.101 \cdots)_{2}$, then $\sigma(2)=3, \sigma(3)=4$, $\sigma(4)=2$.

For $i=2,3, \cdots, n$, we denote the binary form of $C_{\sigma^{-1}(i)}$ by $\left(0 . d_{i 1} d_{i 2} d_{i 3} \cdots\right)_{2} \cdot\left(\sigma^{-1}\right.$ is the inverse of $\sigma$.) Then $Z \leq n$ has the same probability as the following event: in the sequence of bits $d_{21}, d_{31}, \cdots, d_{n 1}$, there is a 0 that appears behind a 1 . We can see that even if the permutation $\sigma$ is arbitrarily fixed, $d_{21}, d_{31}, \cdots, d_{n 1}$ are still $n-1$ i.i.d. random variables with the equal probability of being 0 or 1 , because the permutation $\sigma$ has nothing to do with those bits.

We define the following game: "throw a fair coin at discrete times $1,2,3 \cdots$; if at time $i$, the coin shows HEADS for the first time, then we let $x=i$; if at time $j(j>i)$, the coin shows TAILS for the first time after time $i$, then we let $y=j-i$." By the previous analysis, we see that $\operatorname{Pr}\{Z \leq n\}=\operatorname{Pr}\{x+y \leq$ $n-1\}$. So for any $m, \operatorname{Pr}\{Z=m\}=\operatorname{Pr}\{x+y+1=m\}$. So $E(Z)=E(x+y+1)$ and $\operatorname{Var}(Z)=\operatorname{Var}(x+y+1) . x$ and $y$ both have the geometric distribution and they are independent. So $E(x)=E(y)=\operatorname{Var}(x)=\operatorname{Var}(y)=1 /\left(\frac{1}{2}\right)=2$. So $E(Z)=E(x)+E(y)+1=5$ and $\operatorname{Var}(Z)=\operatorname{Var}(x)+$ $\operatorname{Var}(y)=4$.

\section{Coverage Radius Analysis}

The following result presents the distribution of coverage radius for Algorithm I, when the nodes are deployed on the plane with a Poisson point process of density $\lambda=1$ node per unit area. When $\lambda$ takes general values, the corresponding result can be easily obtained by a simple scaling of the distance measure.

Theorem 3: Assume that nodes are deployed on the plane following a Poisson point process of density $\lambda=1$ node per unit area. Let $R$ denote the coverage radius of a node in a network constructed using Algorithm I. Then for $x \geq 0$, the cumulative distribution function of $R$ is

$$
F_{R}(x)=\operatorname{Pr}\{R \leq x\}=1-e^{-\pi x^{2}}-\pi x^{2} e^{-\frac{\pi x^{2}}{2}} .
$$

For $x \geq 0$, the probability density function of $R$ is $f_{R}(x)=\frac{d F_{R}(x)}{d x}=2 \pi x e^{-\pi x^{2}}+\pi^{2} x^{3} e^{-\frac{\pi x^{2}}{2}}-2 \pi x e^{-\frac{\pi x^{2}}{2}}$.

The expectation and the variance of $R$ are $E(R)=\frac{\sqrt{2}+1}{2} \approx$ 1.207 and $\operatorname{Var}(R)=\frac{5}{\pi}-\frac{3+2 \sqrt{2}}{4} \approx 0.1344$.

Proof: Let $u$ be a generic node with degree $Z$ and coverage radius $R$. Let $n$ denote the number of nodes (except $u$ itself) whose Euclidean distance to $u$ is less than or equal to $x$. The $n$ rays starting at $u$ and respectively going through the $n$ nodes within distance $x$ cut the plane into $n$ cones, whose angle degrees are denoted by $\phi_{1}, \phi_{2}, \cdots, \phi_{n}$. Then, $F_{R}(x)=$ $\operatorname{Pr}\{R \leq x\}=\operatorname{Pr}\{Z \leq n\}=\operatorname{Pr}\left\{\forall 1 \leq i \leq n, \phi_{i}<\pi\right\}=$ $\sum_{j=3}^{\infty} \operatorname{Pr}\{n=j\} \operatorname{Pr}\left\{\forall 1 \leq i \leq n, \phi_{i}<\bar{\pi} \mid n=j\right\}$.

$n$ is a Poisson variable, so $\operatorname{Pr}\{n=j\}=\frac{\left(\pi x^{2}\right)^{j}}{j !} e^{-\pi x^{2}}$. If $u$ is seen as the origin, then for each ray starting at $u$ and going through one of the $n$ nodes that are within distance $x$ from $u$, its absolute angle has an i.i.d. uniform distribution in the range $[0,2 \pi)$. Once the value of $n$ is given, the event " $\forall 1 \leq i \leq n$, 
$\phi_{i}<\pi$ " becomes independent of $x$. Therefore, by Theorem 1 , $\operatorname{Pr}\left\{\forall 1 \leq i \leq n, \phi_{i}<\pi \mid n=j\right\}=1-\frac{j}{2^{j-1}}$. So $F_{R}(x)=$ $\sum_{j=3}^{\infty} \frac{\left(\pi x^{2}\right)^{j}}{j !} e^{-\pi x^{2}}\left(1-\frac{j}{2^{j-1}}\right)=1-e^{-\pi x^{2}}-\pi x^{2} e^{-\frac{\pi x^{2}}{2}}$. The rest of the proof is straightforward.

Theorem 3 shows that the coverage radius on average has a small constant value. It indicates, at the same time, that the distribution of the coverage radius is unbounded. But that is necessary for an infinitely large network since we want no node to be isolated. The positive side is that the percentage of nodes with large coverage radii diminishes exponentially fast to 0 with respect to their coverage radii. If an upper bound is forced onto the coverage radius, then the best one can hope for is to maintain as strong connectivity in the network as possible. Some discussion on this topic is addressed in the concluding remarks.

\section{Routing Property Analysis}

In a network constructed by Algorithm I, when a node $u$ needs to route a message to a destination node $v$, there must exist a node $w$ such that there is a directed edge from $u$ to $w$ and $\angle w u v<\frac{\pi}{2}$. If $d(u, v)=\infty$, then $d(w, v)<d(u, v)$, which implies that by relaying the message to $w, u$ makes the message geographically closer to the destination. For a general destination, the farther away it is, the more likely that $u$ will have a neighbor closer to it. In this sense, the network facilitates geographical routing without dead-ends with high probability for faraway destinations.

\section{E. Connectivity Property Analysis}

Theorem 4: A network constructed using Algorithm I contains a unique infinitely-large and strongly-connected subgraph that every node can reach through directed paths.

Proof: A network constructed by Algorithm I is a directed graph. We use component to denote a maximal strongly connected subgraph. A directed graph must contain one or more components with the following property: a node in the component cannot reach any node outside this component. Such components are called sink components. Every node in a direct graph can reach at least one sink component through directed paths. In the following we first show that every sink component in the network constructed by Algorithm I must be infinitely large, then show that there can exist only one sink component, thus finishing the proof.

Assume that there is a sink component that contains only a finite number of nodes. Consider the convex hull of its nodes on the two-dimensional plane. For every node of the component on the convex hull, one of its cone angles must be no less than $\pi$ since it cannot reach any node outside the convex hull, which contradicts Algorithm I. Therefore every sink component contains infinitely many nodes.

Now assume that there are two sink components $A$ and $B$ of the following property: no edge in $A$ intersects any edge in $B$. Then there exists a curve $S$ on the two-dimensional plane that completely separates $A$ and $B$. For a point $p$ on an edge of $A$, we say that the curve $S$ can see $p$ if there is another point $q$ on $S$ such that a curve segment can connect $p$ and $q$ without intersecting any edge of $A$. The points on the edges of $A$ that $S$ can see is the hull of the nodes in $A$ (respective to $S$ ) composed of line segments. By Algorithm I, each outer angle of this hull (which is toward the curve $S$ ) is less than $\pi$, so the hull bents toward $S$ at each of its turning points. Similarly, the hull of $B$ also bents toward $S$ at each of its turning points, but from the other side of $S$. Then it is impossible that the edges of $A$ and $B$ don't intersect, which contradicts the initial assumption. So for any two sink components $A$ and $B$, there exists a directed edge $u_{1} \rightarrow u_{2}$ in $A$ and a directed edge $v_{1} \rightarrow v_{2}$ in $B$ such that they intersect at a point $t$. Without loss of generality, $d\left(t, u_{2}\right) \geq d\left(t, v_{2}\right)$, then $d\left(u_{1}, u_{2}\right)=d\left(u_{1}, t\right)+d\left(t, u_{2}\right) \geq d\left(u_{1}, t\right)+d\left(t, v_{2}\right) \geq$ $d\left(u_{1}, v_{2}\right)$, which means there is a directed edge from $u_{1}$ to $v_{2}$. That contradicts the definition of sink component. Therefore there is only one sink component in the network constructed by Algorithm I.

Theorem 4 implies that the infinitely many nodes in the unique sink-component can have effective communication among themselves, and that every other node can at least send messages to them (but not receiving messages from them).

\section{Topology Control Algorithm II}

\section{A. Definition of Algorithm II}

The second topology control algorithm, Algorithm II, consists of two steps. It is defined as follows.

Definition 2: In the first step, every node chooses its coverage radius to be the minimum value, subject to the constraint that its cone-angles are all smaller than $\pi$. In the second step, for each node $v$, if its coverage radius was chosen to be $r$ and the length of its longest incoming edge was $t$ in the first step, then $v$ makes its coverage radius to be $\max \{r, t\}$. This topology control method is denoted by Algorithm II.

In other words, given the same set of wireless nodes on the two-dimensional plane, if Algorithm I creates an edge between two nodes, no matter if the edge is unidirectional or bidirectional, Algorithm II creates a bidirectional edge between them.

An example of Algorithm II is shown in Fig. 5.

\section{B. Analysis of Connectivity and Routing Properties}

The routing and connectivity properties of Algorithm I, analyzed in the previous section, are also achieved by Algorithm II, because the edges created by Algorithm II are a superset of the edges created by Algorithm I. Additionally, Algorithm II guarantees the strong-connectivity of the network.

Theorem 5: A network constructed using Algorithm II is strongly connected. 
(a)

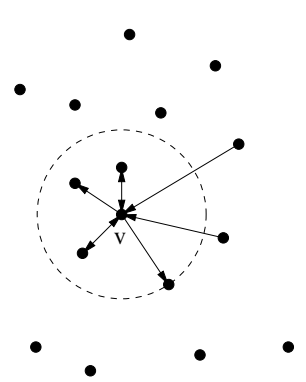

(b)

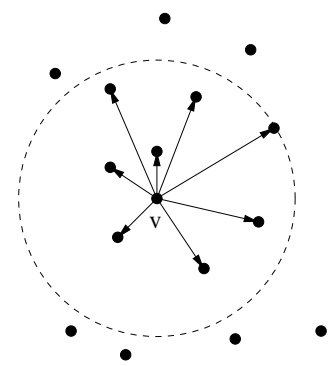

Fig. 5. The nodes in (a) and (b) have the same positions. Assume that when Algorithm I is used for topology control, all the edges incident to $v$ (including both outgoing and incoming edges) are as shown in (a). Then when Algorithm II is used for topology control, all $v$ 's outgoing edges are as shown in (b).

Proof: For the same wireless nodes on the twodimensional plane, Algorithm I and Algorithm II can each create a network, which we denote by Network I and Network II, respectively. For any two nodes $p$ and $q$, if there is a directed path from $p$ to $q$ in Network I, then in Network II, since all the edges in that path become bi-directional, $p$ and $q$ can reach each other through directed paths. Consider two arbitrary nodes $u$ and $v$. By Theorem 4 , in Network I there is a strongly-connected subgraph that $u_{1}$ and $u_{2}$ can both reach. Thus in Network II, $u_{1}$ and $u_{2}$ can reach each other through that subgraph. So Network II is strongly connected.

\section{Coverage Radius Analysis}

For Algorithm II, the distribution of the coverage radius is difficult to compute. Nevertheless, we can still prove that its expectation and variance are upper bounded by small constants, assuming that the nodes are deployed following a Poisson point process with density $\lambda=1$.

Lemma 1: Assume that nodes are deployed on the plane following a Poisson point process of density $\lambda=1$ node per unit area. In a network constructed using Algorithm I, randomly and uniformly select an edge, and denote its length by $L$. (Note that a bi-directional edge is seen as two directed edges of opposite directions.) Then the probability density function of $L$ is

$$
f_{L}(x)=\frac{2 \pi}{5} x\left(e^{-\pi x^{2}}+\pi x^{2} e^{-\frac{\pi x^{2}}{2}}\right),
$$

for $x \geq 0$.

Proof: Let $u$ be a generic node in the network constructed by Algorithm I, and consider its outgoing edges. Use $\Delta(x)$ to denote the probability of the following event: " $u$ has an outgoing edge whose length is between $x$ and $x+d x$," where $d x \rightarrow 0$. Use $n$ to denote the number of nodes within distance $x$ from $u$ (except $u$ itself). The rays starting at $u$ and respectively going through those $n$ nodes cut the plane into $n$ cones, whose angles we denote by $\theta_{1}, \theta_{2}, \cdots, \theta_{n} . \Delta(x)$ is also the probability of having the following two events both happen: event 1 is "there is some $\theta_{i}$ no less than $\pi$," and event 2 is "there is a node whose Euclidean distance to $u$ is between $x$ and $x+d x$." Event 1 and event 2 are independent. Event 1

happens if and only if the coverage radius of $u$ is greater than $x$, so by Theorem 3 , its probability is $e^{-\pi x^{2}}+\pi x^{2} e^{-\frac{\pi x^{2}}{2}}$. The probability of event 2 is $\pi(x+d x)^{2}-\pi x^{2}=2 \pi x d x$ due to the property of Poisson process. So $\Delta(x)=\left(e^{-\pi x^{2}}+\right.$ $\left.\pi x^{2} e^{-\frac{\pi x^{2}}{2}}\right) 2 \pi x d x$.

Theorem 2 shows that the average node degree equals 5. So the summation of $\Delta(x)$ over different values of $x$ equals 5. Randomly and uniformly selecting an edge from the network has the same effect as randomly and uniformly selecting an outgoing edge of $u$, since $u$ is a generic node. So $f_{L}(x)=\left[\lim _{d x \rightarrow 0} \frac{\Delta(x)}{d x}\right] \cdot \frac{1}{5}=\left(e^{-\pi x^{2}}+\pi x^{2} e^{-\frac{\pi x^{2}}{2}}\right) \cdot 2 \pi x \cdot \frac{1}{5}=$ $\frac{2 \pi}{5} x\left(e^{-\pi x^{2}}+\pi x^{2} e^{-\frac{\pi x^{2}}{2}}\right)$, for $x \geq 0$.

Theorem 6: Assume that nodes are deployed on the plane following a Poisson point process of density $\lambda=1$ node per unit area. Let $R$ denote the coverage radius of a node in a network constructed using Algorithm II. Then $E(R) \leq$ 1.6585 , and $\operatorname{Var}(R) \leq 1.338$.

Proof: Imagine that we have a very large bag, and let's play the following game: "deploy nodes on a plane following the Poisson point process of density $\lambda=1$, and use Algorithm I to construct a network; corresponding to each node $u$ of the network, we put a red stick whose length equals the coverage radius of $u$ into the bag; corresponding to each directed edge of the network, we put a green stick whose length equals the length of the edge into the bag; repeat all the above steps."

Clearly there will be infinitely many sticks in the bag, because each network has infinite nodes and we generate the network infinitely many times. Nevertheless, if we use $L_{R}$ to denote the length of a uniformly sampled red stick and use $L_{G}$ to denote the length of a uniformly sampled green stick, we know the probability density functions of $L_{R}$ and $L_{G}: f_{L_{R}}(x)=2 \pi x e^{-\pi x^{2}}+\pi^{2} x^{3} e^{-\frac{\pi x^{2}}{2}}-2 \pi x e^{-\frac{\pi x^{2}}{2}}$ (by Theorem 3), and $f_{L_{G}}(x)=\frac{2 \pi}{5} x\left(e^{-\pi x^{2}}+\pi x^{2} e^{-\frac{\pi x^{2}}{2}}\right)$ (by Lemma 1). Also, Theorem 2 tells us that the average degree of a node is 5 , so the number of green sticks is 5 times the number of red sticks. Therefore, if we use $L$ to denote the length of a uniformly sampled stick in the bag (without considering its color), then the probability density function of $L$ is $f_{L}(x)=$ $\left[f_{L_{R}}(x)+5 f_{L_{G}}(x)\right] \cdot \frac{1}{6}=\frac{2 \pi}{3} x e^{-\pi x^{2}}+\frac{\pi^{2}}{2} x^{3} e^{-\frac{\pi x^{2}}{2}}-\frac{\pi}{3} x e^{-\frac{\pi x^{2}}{2}}$.

For Algorithm II, we see (from its definition) that a node determines its coverage radius through these two steps: "firstly, Algorithm I is used to construct a network; next, every node sets its coverage radius to be the larger value among its old coverage radius and the length of its longest old incoming edge." Equivalently, we can also see the nodes as using the following method to determine their coverage radii: "every node picks a red stick and a green stick (not necessarily uniformly) from the bag, and sets its coverage radius to be the maximum length of these two sticks." Clearly, no two nodes should pick the same red stick or green stick.

We can see that the average coverage radius for Algorithm II cannot exceed the average coverage radius for the following scheme: "pick just one stick out of the bag for each node (one 
by one), and set the node's coverage radius to be equal to that stick's length; for every node, we always pick the longest stick that is still available (regardless of its color)." What is the average length of the sticks picked out in the above scheme? The number of sticks in the bag is 6 times the number of nodes. So if we use $z$ to denote the length of the shortest stick that is picked, then $\int_{z}^{\infty} f_{L}(x) d x=\frac{1}{6}-$ by this relationship, we find $z \approx 1.4115$. Then the average length of the sticks picked out in the above scheme is $\left[\int_{z}^{\infty} x f_{L}(x) d x\right] \cdot 6=1.6585$. So $E(R) \leq 1.6585$.

With the same method we can prove that $E\left(R^{2}\right) \leq 2.7948$. The average coverage radius for Algorithm II is no less than that of algorithm I, so $E(R) \geq \frac{\sqrt{2}+1}{2} \approx 1.207$ (by Theorem 3 ). So $\operatorname{Var}(R)=E\left(R^{2}\right)-E^{2}(R) \leq 2.7948-1.207^{2}=1.338$.

\section{Topology CONTROL Algorithm III}

\section{A. Definition of Algorithm III}

In the following topology control algorithm, the threshold on cone angles is reduced to be less than $\pi$. In specific, it is set to be a parameter $\theta$, where $\theta<\frac{2 \pi}{3}$. Reducing the threshold on cone angles strengthens the connectivity of the network. As a result, more efficient routing paths are created.

Definition 3: Let $\theta$ be a fixed number where $0<\theta<\frac{2 \pi}{3}$. Every node chooses its coverage radius to be the minimum value, subject to the constraint that its cone-angles are all smaller than or equal to $\theta$. This topology control method is denoted by Algorithm III.

\section{B. Analysis of Connectivity and Routing Properties}

Lemma 2: Let $u$ and $v$ be two nodes in a network constructed using Algorithm III. If there is no directed edge from $u$ to $v$, there must exist a node $w$, such that $d(w, v)<d(u, v)$ and there is a directed edge from $u$ to $w$.

Proof: Let $r$ be the coverage radius of $u$. If there is no directed edge from $u$ to $v$, then $d(u, v)>r$. Since all $u$ 's cone-angles are smaller than or equal to $\theta, u$ must have an outgoing edge to a neighboring node $w$ such that the angle $\angle w u v \leq \frac{\theta}{2}<\frac{\pi}{3}$. Then since $d(u, w) \leq r<d(u, v)$, we get $d(w, v)<d(u, v)$.

If in Algorithm III we make $\theta>\frac{2 \pi}{3}$, then the statement in Lemma 2 will no longer hold (however it will hold if $\theta=\frac{2 \pi}{3}$ ). So in this sense, $\frac{2 \pi}{3}$ is a threshold for $\theta$ in order to get the property stated in Lemma 2 . From the above lemma, it can be seen that for any source node and destination node, the greedy geographical routing can find a routing path between them without meeting any dead-end. Thus the following claim is true.

Theorem 7: A network constructed using Algorithm III is strongly connected. What's more, it enables geographical routing with no dead-ends for any source-destination node pair.

\section{Analysis of Length Distortion and Routing Properties}

In this subsection, we prove that in a network constructed by Algorithm III, the length distortion for any node pair is upper bounded. In addition, a simple geographical routing method can achieve a bounded length distortion as well.

Lemma 3: Let $O$ and $P$ be two nodes, and let $O A E$ be a sector as shown in Fig. 6, where $0<\angle \mathrm{AOP} \leq \frac{\pi}{3}$ and $d(\mathrm{O}, \mathrm{P})>$ $d(\mathrm{O}, \mathrm{A})=d(\mathrm{O}, \mathrm{E})$. For every point $t$ in the sector (including the boundary of the sector), we define $\eta(t)$ as

$$
\eta(t)=\frac{d(\mathrm{O}, t)}{d(\mathrm{O}, \mathrm{P})-d(t, \mathrm{P})},
$$

and call $\eta(t)$ the competitive ratio of $t$. Then, in the sector, $A$ is the unique point that maximizes the competitive ratio and that maximum value, $\eta(\mathrm{A})$, is

$$
\frac{d(\mathrm{O}, \mathrm{A})}{d(\mathrm{O}, \mathrm{P})-\sqrt{d^{2}(\mathrm{O}, \mathrm{P})+d^{2}(\mathrm{O}, \mathrm{A})-2 d(\mathrm{O}, \mathrm{P}) d(\mathrm{O}, \mathrm{A}) \cos \angle \mathrm{AOP}}} .
$$

$\eta(\mathrm{A})$ is a strictly decreasing function in $d(\mathrm{O}, \mathrm{P})$.

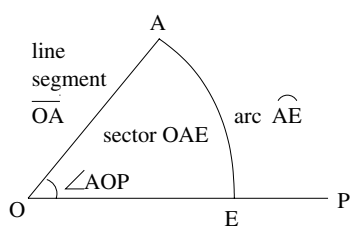

Fig. 6. Maximum competitive ratio in a sector.

Proof: Let $C$ be a circle centered at $P$ that intersects the sector. If we move a point $c$ along $C$, the value $d(\mathrm{O}, \mathrm{P})-d(c, \mathrm{P})$ remains constant. If $C$ intersects the arc $\widehat{\mathrm{AE}}$, then for points on $C$ that are also in the sector, the intersection of $C$ and $\widehat{\mathrm{AE}}$ is the only point that maximizes the competitive ratio. If $C$ does not intersect the arc $\widehat{\mathrm{AE}}$, then it intersects the line segment $\overline{\mathrm{OA}}$; then it's also clear that for points on $C$ that are also in the sector, the intersection of $C$ and $\overline{\mathrm{OA}}$ is the only point that maximizes the competitive ratio. So the point (or points) in the sector that maximizes the competitive ratio is either on the arc $\widehat{\mathrm{AE}}$ or on the line segment $\overline{\mathrm{OA}}$. The formula of the competitive ratio for points on $\widehat{\mathrm{AE}}$ and $\overline{\mathrm{OA}}$ can be derived with simple calculation, and its maximum value can be found using simple algebraic methods. As a result, we find that the maximum competitive ratio is achieved only for the point A. The formula for $\eta(\mathrm{A})$ in this lemma can be easily seen to be correct. $\eta(\mathrm{A})$ is a strictly decreasing function in $d(\mathrm{O}, \mathrm{P})$ if and only if $d(\mathrm{O}, \mathrm{P})-d(\mathrm{~A}, \mathrm{P})=$ $d(\mathrm{O}, \mathrm{P})-\sqrt{d^{2}(\mathrm{O}, \mathrm{P})+d^{2}(\mathrm{O}, \mathrm{A})-2 d(\mathrm{O}, \mathrm{P}) d(\mathrm{O}, \mathrm{A}) \cos \angle \mathrm{AOP}}$ is a strictly increasing function in $d(\mathrm{O}, \mathrm{P})$. Then since the derivative of $d(\mathrm{O}, \mathrm{P})-d(\mathrm{~A}, \mathrm{P})$ in $d(\mathrm{O}, \mathrm{P})$ equals

$$
1-\frac{2 d(\mathrm{O}, \mathrm{P})-2 d(\mathrm{O}, \mathrm{A}) \cos \angle \mathrm{AOP}}{2 \sqrt{d^{2}(\mathrm{O}, \mathrm{P})+d^{2}(\mathrm{O}, \mathrm{A})-2 d(\mathrm{O}, \mathrm{P}) d(\mathrm{O}, \mathrm{A}) \cos \angle \mathrm{AOP}}},
$$

which equals

$$
1-\frac{d(\mathrm{O}, \mathrm{P})-d(\mathrm{O}, \mathrm{A}) \cos \angle \mathrm{AOP}}{\sqrt{[d(\mathrm{O}, \mathrm{P})-d(\mathrm{O}, \mathrm{A}) \cos \angle \mathrm{AOP}]^{2}+d^{2}(\mathrm{O}, \mathrm{A}) \sin ^{2} \angle \mathrm{AOP}}},
$$


we see that the value is greater than 0 . So $\eta(\mathrm{A})$ is strictly decreasing in $d(\mathrm{O}, \mathrm{P})$.

The following simple geographical routing method is guaranteed to work in a network constructed using Algorithm III: "when a node $u$ needs to forward a message to a destination node $v, u$ sends the message to a neighboring node $w$ such that $\angle w u v \leq \frac{\theta}{2}<\frac{\pi}{3}$ if $v$ is not a neighboring node of $u$." We call it Routing Method I. Now we prove the result on length distortions.

Theorem 8: In a network constructed using Algorithm III, for any two nodes $u$ and $v$, the length distortion for the ordered node pair $(u, v)$ is upper bounded by

$$
\frac{1}{1-\sqrt{2-2 \cos \frac{\theta}{2}}} \text {. }
$$

Proof: In Lemma 3, it is stated that $\eta(\mathrm{A})$ is strictly decreasing in $d(\mathrm{O}, \mathrm{P})$, so $\eta(\mathrm{A})$ is upper bounded by

$$
\frac{d(\mathrm{O}, \mathrm{A})}{d(\mathrm{O}, \mathrm{A})-\sqrt{d^{2}(\mathrm{O}, \mathrm{A})+d^{2}(\mathrm{O}, \mathrm{A})-2 d(\mathrm{O}, \mathrm{A}) d(\mathrm{O}, \mathrm{A}) \cos \angle \mathrm{AOP}}},
$$

which equals

$$
\frac{1}{1-\sqrt{2-2 \cos \angle \mathrm{AOP}}} \text {. }
$$

Now for any two nodes $u$ and $v$ in the network constructed by Algorithm III, we use Routing Method I to get a path from $u$ to $v$. Let's say that the path consists of nodes $u=w_{1}, w_{2}$, $w_{3}, \cdots, w_{k+1}=v$. Then the length distortion for the ordered node pair $(u, v)$ is at most

$$
\frac{\sum_{i=1}^{k} d\left(w_{i}, w_{i+1}\right)}{d\left(w_{1}, w_{k+1}\right)},
$$

which equals

$$
\frac{\sum_{i=1}^{k} d\left(w_{i}, w_{i+1}\right)}{\sum_{i=1}^{k}\left[d\left(w_{i}, w_{k+1}\right)-d\left(w_{i+1}, w_{k+1}\right)\right]} .
$$

For $i=1,2, \cdots, k, \angle w_{i+1} w_{i} w_{k+1} \leq \frac{\theta}{2}<\frac{\pi}{3}$, and the value of

$$
\frac{d\left(w_{i}, w_{i+1}\right)}{d\left(w_{i}, w_{k+1}\right)-d\left(w_{i+1}, w_{k+1}\right)}
$$

is upper bounded by

$$
\frac{1}{1-\sqrt{2-2 \cos \angle w_{i+1} w_{i} w_{k+1}}},
$$

which is in turn upper bounded by

$$
\frac{1}{1-\sqrt{2-2 \cos \frac{\theta}{2}}} \text {. }
$$

Therefore the length distortion for the ordered node pair $(u, v)$ is at most $\frac{1}{1-\sqrt{2-2 \cos \frac{\theta}{2}}}$.

The proof of Theorem 8 actually shows that even by just using Routing Method I, a geographical routing scheme, the ratio between the routing path's length and the Euclidean distance between two arbitrarily chosen source and destination nodes is still upper bounded by $\frac{1}{1-\sqrt{2-2 \cos \frac{\theta}{2}}}$.

\section{Analysis of Hop Distortion, Node Degree and Coverage Radius}

For a network constructed using Algorithm III, the hop distortion for an ordered node pair can be arbitrarily large. That is because the node pair may exist in an area where the node density is very high, and as a result, every path connecting those two nodes consists of too many edges of very small lengths. In that case, even if the length distortion for the node pair is bounded, the hop distortion can still be unbounded.

When the nodes are assumed to follow a Poisson point process of density $\lambda$, the distributions of the node degree and the coverage radius can be computed in a similar way as that for Algorithm I. We comment, however, that it appears difficult to obtain a closed-form expression when the parameter $\theta$ takes the general value. It is not difficult to see that for Algorithm III, like for Algorithm I, the probability density functions for node degree and coverage radius both decrease exponentially fast as the values of node degree and coverage radius increase. Thus the expectations and variances of node degree and coverage radius have finite values (while considering the parameters $\theta$ and $\lambda$ as fixed numbers).

\section{Topology Control Algorithm IV}

\section{A. Definition of Algorithm IV}

The fourth topology control algorithm, Algorithm IV, sets a lower bound $\delta$ for the coverage radius. It consists of two steps, and is defined as follows.

Definition 4: In the first step, every node chooses its coverage radius to be the minimum value, subject to the constraint that its cone-angles are all smaller than or equal to $\theta$, where $\theta$ is a given parameter and $0<\theta<\frac{2 \pi}{3}$. In the second step, for those nodes whose coverage radii were smaller than $\delta$ in the first step, they set their coverage radii to be $\delta$. Here $\delta$ is a given parameter and $\delta>0$. This topology control method is denoted by Algorithm IV.

\section{B. Hop Distortion Analysis}

For the same wireless nodes on a two-dimensional plane, the network constructed by Algorithm IV contains the network constructed by Algorithm III as a subgraph. So for a network constructed using Algorithm IV, it is also strongly connected, and the upper bound on length distortion holds here, too. Now we show that Algorithm IV can achieve more: the hop distortion for any node pair is upper-bounded.

Firstly we present a greedy routing algorithm, which we shall call Routing Method II, as follows: "when a node $u$ needs to forward a message to a destination node $v$, if there is no edge from $u$ to $v, u$ sends the message to its neighbor $w$ that satisfies the following two conditions: (1) define $S$ as $S=\{p$ $\mid u$ has an outgoing edge to $p$, and $\left.\angle p u v \leq \frac{\theta}{2}<\frac{\pi}{3}\right\}$, then $w \in S$; (2) $\forall p \in S, d(w, v) \leq d(p, v)$." Routing method II is a special case of Routing Method I. 
Lemma 4: In a network constructed by Algorithm IV, if Routing Method II is used, then for any two consecutive edges in any routing path, the total length of those two edges is greater than $\delta$.

Proof: Assume Routing Method II is used. Consider the following scenario: "node $\mathrm{O}$ needs to forward a message to destination node $\mathrm{P}$, and it sends the message to its neighbor C; node $\mathrm{C}$ sends the message to its neighbor D." Then to prove this lemma, we need to prove that $d(\mathrm{O}, \mathrm{C})+d(\mathrm{C}, \mathrm{D})>\delta$. (a)

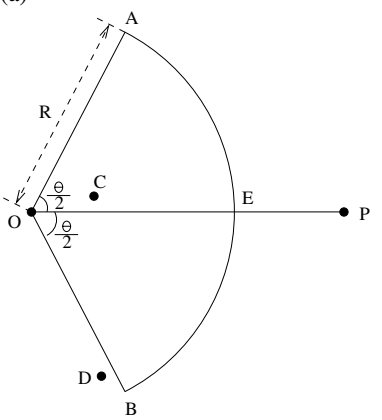

(c)

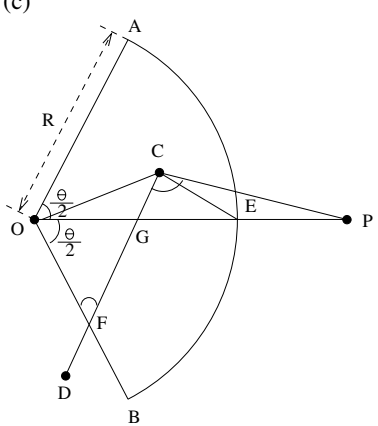

(e)

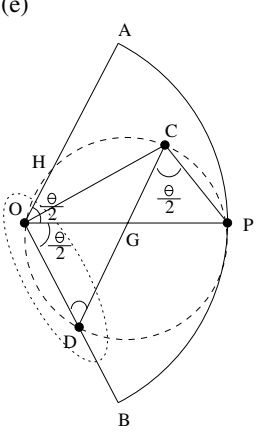

(b)

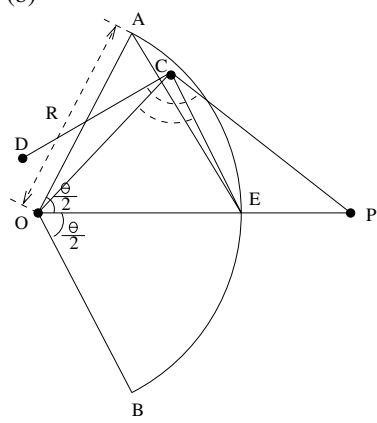

(d)

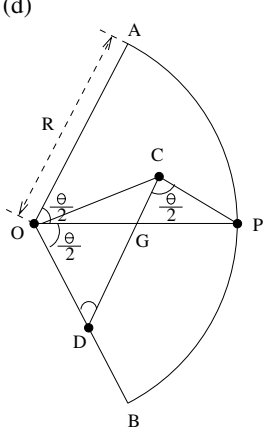

other nodes in the sector $\mathrm{OAB}$, D must be out of the sector $\mathrm{OAB}$. Then there are three possibilities: Possibility 1, the line segment $\overline{\mathrm{CD}}$ intersects $\overline{\mathrm{OA}}$; Possibility $2, \overline{\mathrm{CD}}$ intersects the arc $\widehat{\mathrm{AB}}$; Possibility 3, $\overline{\mathrm{CD}}$ intersects $\overline{\mathrm{BO}}$. We consider those three possibilities. Without loss of generality, from now on assume node $\mathrm{C}$ is in the sector OAE, where $\mathrm{E}$ is the point where the line segment $\overline{\mathrm{OP}}$ and the arc $\widehat{\mathrm{AB}}$ intersects.

(1) Firstly, assume $\overline{\mathrm{CD}}$ intersects $\overline{\mathrm{OA}}$. Then it is as shown in Fig. 7 (b). This is in fact an impossible case.

Imagine there is a circle that passes through these three points - $\mathrm{O}$, A and $\mathrm{E}$ - which we shall denote by 'circle OAE'. All the three inner angles in the triangle $\triangle \mathrm{OAE}$ are smaller than $\frac{\pi}{2}$, so the center of the 'circle OAE' is inside the triangle $\triangle \mathrm{OAE}$ - so the sector OAE is totally contained inside the 'circle OAE'; then since node $\mathrm{C}$ lies in the sector OAE, $\mathrm{C}$ is in the 'circle $\mathrm{OAE}$ ' - so $\angle \mathrm{OCE} \geq \mathrm{OAE}$. Then $\angle \mathrm{DCP} \geq \angle \mathrm{OCE} \geq$ $\angle \mathrm{OAE}=\angle \mathrm{OEA}=\frac{\pi-\angle \mathrm{AOE}}{2}=\frac{\pi-\frac{\theta}{2}}{2}>\frac{\pi-\frac{2 \pi / 3}{2}}{2}=\frac{\pi}{3}>\frac{\theta}{2}$. Also, $2 \pi-\angle \mathrm{DCP} \geq \angle \mathrm{ACP}>\angle \mathrm{AOP}=\frac{\theta}{2}$. That contradicts the fact that $\mathrm{C}$ decided to send the message to $\mathrm{D}$ based on the Routing Method II.

(2) Secondly, assume $\overline{\mathrm{CD}}$ intersects the arc $\widehat{\mathrm{AB}}$. In this case, clearly $d(\mathrm{O}, \mathrm{C})+d(\mathrm{C}, \mathrm{D}) \geq d(\mathrm{O}, \mathrm{D})>R \geq \delta$, which is the conclusion we need.

(3) Thirdly, assume $\overline{\mathrm{CD}}$ intersects $\overline{\mathrm{OB}}$. Then it is as shown in Fig. 7 (c).

Let's see how we can adjust the positions of all the nodes to minimize the value of $d(\mathrm{O}, \mathrm{C})+d(\mathrm{C}, \mathrm{D})$. The following are the 2 steps for that adjustment: "(i) Make node D to be infinitely close to the point $\mathrm{F}$. (ii) We know $\angle \mathrm{PCF}=\angle \mathrm{PCD} \leq \frac{\theta}{2}<$ $\frac{\pi}{3}$, so $\angle \mathrm{OFC}=\pi-\angle \mathrm{FOP}-\angle \mathrm{OGF} \leq \pi-\angle \mathrm{PCF}-\angle \mathrm{CGP}=$ $\angle \mathrm{CPO}<\angle \mathrm{CEO}<\frac{\pi}{2}$. So to minimize the value of $d(\mathrm{C}, \mathrm{F})$ (which now equals $d(\mathrm{C}, \mathrm{D})$ ), we need to make $\angle \mathrm{PCF}=\frac{\theta}{2}$, and make node $\mathrm{P}$ be infinitely close to the point E." After the above two adjustment steps, the situation becomes as shown in Fig. 7 (d).

Now let's temporarily assume that the position of node D is fixed, and let's see where node $\mathrm{C}$ needs to be in order to minimize $d(\mathrm{O}, \mathrm{C})+d(\mathrm{C}, \mathrm{D})$. Imagine there is a circle that passes through these three nodes $-\mathrm{O}, \mathrm{P}$ and $\mathrm{D}-$ which we shall call 'circle OPD'. (Circle OPD is the dashed circle in Fig. 7 (e).) Since $\angle \mathrm{POD}=\angle \mathrm{PCD}=\frac{\theta}{2}$, C must be on the circle OPD. C is also in the sector OAP, so node $\mathrm{C}$ can only lie on the arc $\widehat{\mathrm{HP}}$. (The arc $\widehat{\mathrm{HP}}$ is part of the circle OPD, and $\mathrm{H}$ is the intersection point of $\overline{\mathrm{OA}}$ and the circle OPD.) But at which position on the arc $\widehat{\mathrm{HP}}$ should node $\mathrm{C}$ be in order to minimize $d(\mathrm{O}, \mathrm{C})+d(\mathrm{C}, \mathrm{D})$ ?

On the two-dimensional plane, given any number $s$ such that $s>d(\mathrm{O}, \mathrm{D})$, if we find out those points $p$ such that $d(\mathrm{O}, p)+$ $d(p, \mathrm{D})=s$, then we'll see that those points form an ellipse with $O$ and $D$ as its foci. (Such an ellipse is shown in dotted line in Fig. 7 (e).) Let's gradually increase the value of $s$, and as a result the ellipse grows. Once the ellipse intersects the arc $\widehat{H P}$, the first intersection point is where node $C$ should be in order to minimize $d(\mathrm{O}, \mathrm{C})+d(\mathrm{C}, \mathrm{D})$. Clearly this intersection point is either point $\mathrm{H}$ or point $\mathrm{P}$. If the intersection point is $\mathrm{P}$, then we already have $d(\mathrm{O}, \mathrm{C})+d(\mathrm{C}, \mathrm{D})=d(\mathrm{O}, \mathrm{P})+d(\mathrm{C}, \mathrm{D})>$ $d(\mathrm{O}, \mathrm{P}) \geq \delta$. So in the rest of the proof, we assume that node $d(\mathrm{D}, \mathrm{P})<d(\mathrm{C}, \mathrm{P})$ and $\mathrm{C}$ is the closest to $\mathrm{P}$ than all the the

If $\mathrm{P}$ and $\mathrm{D}$ are the same node, then $d(\mathrm{O}, \mathrm{C})+d(\mathrm{C}, \mathrm{D})>$ $\delta$. That is because O's not sending the message directly to $\mathrm{P}$ implies that $d(\mathrm{O}, \mathrm{P})$ is greater than the coverage radius of $\mathrm{O}$, which is lower bounded by $\delta$. In the following proof, we assume that $\mathrm{P}$ and $\mathrm{D}$ are not the same node.

Let the coverage radius of node o be denoted by $R$. See Fig. 7 (a). Clearly, node $C$ must be in the sector OAB; since 
$C$ lies where point $\mathrm{H}$ is, and we'll prove that even in this case, we still have $d(\mathrm{O}, \mathrm{C})+d(\mathrm{C}, \mathrm{D})>\delta$.

Since now node $\mathrm{C}$ lies where $\mathrm{H}$ is, the situation becomes as shown in Fig. 7 (f). From now on we no longer assume that node D's position is fixed. Instead, we let node $\mathrm{C}$ take different positions on the line segment $\overline{\mathrm{OA}}$; and the position of $\mathrm{D}$ becomes uniquely determined by $\mathrm{C}$.

Define $t$ as $t=d(\mathrm{O}, \mathrm{C})$, and define $f(t)$ as $f(t)=$ $d(\mathrm{O}, \mathrm{C})+d(\mathrm{C}, \mathrm{D})$. (Here $0<t \leq d(\mathrm{O}, \mathrm{A})=R$.) Then $f(t)=d(\mathrm{O}, \mathrm{C})+d(\mathrm{C}, \mathrm{D})=t+\frac{d(\mathrm{C}, \mathrm{D}) \sin \angle \mathrm{CDO}}{\sin \angle \mathrm{CDO}}=t+$ $\frac{d(\mathrm{O}, \mathrm{C}) \sin \angle \mathrm{COD}}{\sin \angle \mathrm{CPO}}=t+\frac{t \sin \theta}{\sin \angle \mathrm{CPO}}=t+\frac{d(\mathrm{C}, \mathrm{P}) \cdot t \sin \theta}{d(\mathrm{C}, \mathrm{P}) \sin \angle \mathrm{CPO}}=$ $t+\frac{d(\mathrm{C}, \mathrm{P}) \cdot t \sin \theta}{d(\mathrm{O}, \mathrm{C}) \sin \angle \mathrm{COP}}=t+\frac{d(\mathrm{C}, \mathrm{P}) \cdot t \sin \theta}{t \sin \frac{\theta}{2}}=t+2 \cos \frac{\theta}{2} d(\mathrm{C}, \mathrm{P})=$ $t+2 \cos \frac{\theta}{2} \sqrt{d^{2}(\mathrm{O}, \mathrm{C})+d^{2}(\mathrm{O}, \mathrm{P})-2 d(\mathrm{O}, \mathrm{C}) d(\mathrm{O}, \mathrm{P}) \cos \angle \mathrm{COP}}=t+$ $2 \cos \frac{\theta}{2} \sqrt{t^{2}+R^{2}-2 t R \cos \frac{\theta}{2}}$.

Let's see when $f(t)$ takes its minimum value. Assume that there is a value $t_{0}\left(0<t_{0} \leq R\right)$ such that the derivative of $f(t)$ at $t=t_{0}-$ namely, $\left.\frac{d f(t)}{d t}\right|_{t=t_{0}}$ - equals 0 . By solving the equation $\frac{d f(t)}{d t}=0$, we find that $t_{0}=R \cos \frac{\theta}{2}-$ $\frac{R \sin \frac{\theta}{2}}{\sqrt{4 \cos ^{2} \frac{\theta}{2}-1}}$. By using the fact that $t_{0}>0$ and the above formula, we find that $\theta<\frac{\pi}{2}$. Then we find that $f\left(t_{0}\right)=$ $R \cos \frac{\theta}{2}+R \sin \frac{\theta}{2} \sqrt{4 \cos ^{2} \frac{\theta}{2}-1}>R \cos \frac{\theta}{2}+R \sin \frac{\theta}{2}=$ $R \sqrt{\cos ^{2} \frac{\theta}{2}+\sin ^{2} \frac{\theta}{2}+2 \cos \frac{\theta}{2} \sin \frac{\theta}{2}}=R \sqrt{1+\sin \theta}>R \geq \delta$. (Note that $0<\theta<\frac{2 \pi}{3}$.) So $f\left(t_{0}\right)>\delta$. Now let's check the boundary values. When $t=d(\mathrm{O}, \mathrm{A})=R$, clearly $f(t)>$ $R \geq \delta$. When $t$ approaches 0 , the value of $f(t)$ approaches $2 R \cos \frac{\theta}{2}>2 R \cos \frac{\pi}{3}=R \geq \delta$. Then it's not difficult to see that $f(t)>\delta$ for any $0<t \leq R$. So the conclusion has been proved in this case.

Theorem 9: In a network constructed by Algorithm IV, use Routing Method II for routing. For any two nodes $\mathrm{O}$ and $\mathrm{P}$, use $H(\mathrm{O}, \mathrm{P})$ to denote the number of edges in the routing path from $\mathrm{O}$ to $\mathrm{P}$. Assume $H(\mathrm{O}, \mathrm{P}) \geq 2$. Then $\frac{H(\mathrm{O}, \mathrm{P})}{d(\mathrm{O}, \mathrm{P})}$ is upper bounded as follows:

$$
\frac{H(\mathrm{O}, \mathrm{P})}{d(\mathrm{O}, \mathrm{P})}<\frac{2}{d(\mathrm{O}, \mathrm{P})}+\frac{2}{\delta\left(1-\sqrt{2-2 \cos \frac{\theta}{2}}\right)} .
$$

Proof: By Theorem 8 and the fact that Routing Method II is a special case of Routing Method I, the length of the routing path from $\mathrm{O}$ to $\mathrm{P}$ is at most $d(\mathrm{O}, \mathrm{P}) \cdot \frac{1}{1-\sqrt{2-2 \cos \frac{\theta}{2}}}$. The total length of any two consecutive edges in the routing path is more than $\delta$ (by Lemma 4), so the first $2\left\lfloor\frac{H(\mathrm{O}, \mathrm{P})-1}{2}\right\rfloor$ edges in the routing path have a total length of more than $\delta\left\lfloor\frac{H(\mathrm{O}, \mathrm{P})-1}{2}\right\rfloor$ - but that total length is less than the length of the routing path. So $\delta\left\lfloor\frac{H(\mathrm{O}, \mathrm{P})-1}{2}\right\rfloor<d(\mathrm{O}, \mathrm{P}) \cdot \frac{1}{1-\sqrt{2-2 \cos \frac{\theta}{2}}}$. As a result, $\frac{H(\mathrm{O}, \mathrm{P})}{d(\mathrm{O}, \mathrm{P})} \leq \frac{2\left\lfloor\frac{H(\mathrm{O}, \mathrm{P})-1}{2}\right\rfloor+2}{d(\mathrm{O}, \mathrm{P})}<\frac{2}{d(\mathrm{O}, \mathrm{P})}+\frac{2}{\delta\left(1-\sqrt{2-2 \cos \frac{\theta}{2}}\right)}$.

In Theorem 9 , clearly $d(\mathrm{O}, \mathrm{P})>\delta$. So we have the following conclusion.
Theorem 10: In a network constructed by Algorithm IV, for any two nodes $u$ and $v$ such that $u$ has no outgoing edge to $v$, the hop distortion for the ordered node pair $(u, v)$ is less than

$$
\frac{2}{\delta}\left(1+\frac{1}{1-\sqrt{2-2 \cos \frac{\theta}{2}}}\right) .
$$

We can see that even by just using Routing Method II, a geographical routing method different from shortest path routing, for any two source and destination nodes such that the source has no outgoing edge to the destination, the ratio of the number of hops in the routing path to the Euclidean distance between the source-destination pair is still upper-bounded by $\frac{2}{\delta}\left(1+\frac{1}{1-\sqrt{2-2 \cos \frac{\theta}{2}}}\right)$.

\section{Analysis of Node Degree and Coverage Radius}

When the nodes follow a Poisson point process of density $\lambda$, the distributions of node degree and coverage radius for Algorithm IV can be computed primarily based on the corresponding distributions for Algorithm III. For Algorithm IV, the probability density functions for node degree and coverage radius also decrease exponentially fast as their values increase. Thus their expectations and variances have finite values (while considering the parameters $\theta, \delta$ and $\lambda$ as fixed numbers).

\section{CONCLUDING REMARKS}

In this paper, we have presented four topology-control algorithms. Their performance is summarized in Table 1, with respect to the seven performance criteria shown in Section I. (A-I, A-II, A-III and A-IV respectively mean Algorithms I, II, III and IV.)

We point out that for those entries labelled as $\Theta(1)$ in Table 1, their values actually depend on the parameters in the corresponding algorithms (namely, $\lambda, \theta$ and $\delta$, if they exist). Since we regard $\lambda, \theta$ and $\delta$ as given (fixed) numbers, the values of the entries can indeed be seen as constants. This should be contrasted with the performance of many previous topology-control algorithms, whose values for these entries can be extremely large. For example, with the scheme that all nodes have the same coverage radius [8], to achieve strong connectivity, both the coverage radius and the average node degree need to be infinity. With the scheme that all nodes have the same node degree [23], to achieve asymptotic strong connectivity, the degree of every node needs to be $\Omega(\log n)$, where $n$ denotes the total number of nodes in the network. Then when $n \rightarrow \infty$, both the average node degree and the average coverage radius become infinity.

The upper bounds on hop distortion and length distortion presented in this paper are for all node pairs. They are independent of the distribution of the node positions. When an appropriate distribution model is assumed, analysis of the average case distortion is also valuable [2] [12]. 


\begin{tabular}{|c|c|c|c|c|}
\hline & $A-I$ & $A-I I$ & $A-I I I$ & $A-I V$ \\
\hline $\begin{array}{c}\text { Localized } \\
\text { construction }\end{array}$ & Yes & Yes & Yes & Yes \\
\hline $\begin{array}{c}\text { Strong } \\
\text { connectivity }\end{array}$ & - & Yes & Yes & Yes \\
\hline $\begin{array}{c}\text { Average } \\
\text { node degree }\end{array}$ & $\Theta(1)$ & - & $\Theta(1)$ & $\Theta(1)$ \\
\hline $\begin{array}{c}\text { Average } \\
\text { coverage } \\
\text { radius }\end{array}$ & $\Theta(1)$ & $\Theta(1)$ & $\Theta(1)$ & $\Theta(1)$ \\
\hline $\begin{array}{c}\text { Hop } \\
\text { distortion } \\
\text { upper bound* }\end{array}$ & $\infty$ & $\infty$ & $\infty$ & $\Theta(1)$ \\
\hline $\begin{array}{c}\text { Length } \\
\text { distortion } \\
\text { upper bound* }\end{array}$ & $\infty$ & - & $\Theta(1)$ & $\Theta(1)$ \\
\hline $\begin{array}{c}\text { Enabling } \\
\text { geographical } \\
\text { routing } \\
\text { with no } \\
\text { dead-ends }\end{array}$ & $\begin{array}{c}\text { Far } \\
\text { only }\end{array}$ & $\begin{array}{c}\text { Far } \\
\text { only }\end{array}$ & Yes & Yes \\
\hline
\end{tabular}

Hop distortion upper bound*, Length distortion upper bound ${ }^{*}$ : these upper bounds are for the maximum hop/length distortion for all the ordered node pairs in the network.

Far only ${ }^{\dagger}$ : geographical routing is enabled with no dead-ends with high probability only for faraway destinations.

Table 1: Summary of performance of the four topology-control algorithms.

The results in this paper can have several topics as their natural extensions. Firstly, the network considered here consists of infinitely many nodes, which is only an approximation of large-scale networks. For networks of finite sizes, an efficient mechanism for dealing with the effect of network boundaries is needed. Secondly, if upper bounds are enforced onto the coverage radii of nodes, then constructing a network topology without isolated nodes become impossible if the node positions follow random point processes like the Poisson point process. In such cases, the topology control algorithms should be modified for more realistic objectives, such as achieving the best possible network connectivity instead of strong connectivity. For example, if we assume that all the nodes have the same upper limit $A$ on their coverage radii, then we can modify our Algorithm III to be the following algorithm (which consists of two steps): "STEP 1, every node uses Algorithm III to determine its coverage radius; STEP II, for every node, if its coverage radius was greater than $A$ in step 1 , then the nodes sets its coverage radius to be $A$." Let's call a network constructed using the above algorithm Network I. To see how well nodes are connected in Network I, let's construct a second network, which we call Network II, in the following way: "every nodes sets its coverage radius to be $A$." (Clearly, the nodes in Network II are connected as strongly as possible.) Then we can prove the following result:

"For any two nodes $u$ and $v$, $u$ can reach $v$ in Network $I$ if and only if $u$ can reach $v$ in Network II."

The proof for the above result is essentially the same as the proof for the Theorem IV.3 in [22]. So we omit its details.

Our four algorithms take different combinations of the three topology-control tools. Based on them, one can also predict the performance of other algorithms using varied combinations of those tools.

Topology control based on angle information is a very promising direction for the design of network topologies that achieve excellent global properties by using just localized constructions. To progress in this direction, we are interested in studying topology-control schemes that are fault-tolerant and more adaptive to transmission power constraints and signal interference. Those remain as our future research topics.

\section{ACKNOWLEDGMENT}

The authors would like to thank Matthew Cook, Jie Gao and Michael Langberg for their helpful discussions, and thank the anonymous reviewers for their helpful comments.

This work was supported in part by the Lee Center for Advanced Networking at the California Institute of Technology, and by NSF grant CCR-TC-0209042.

\section{REFERENCES}

[1] K. Alzoubi, X. Li, Y. Wang, P. Wan and O. Frieder, "Geometric spanners for wireless ad hoc networks," IEEE Trans. Parallel and Distributed Systems, vol. 14, no. 4, pp. 408-421, 2003.

[2] F. Baccelli, B. Blaszczyszyn and P. Muhlethaler, "A spatial reuse Aloha MAC protocol for multihop wireless mobile networks," in Proc. Annual Conf. on Communication, Allerton, USA, 2003.

[3] P. Bose, P. Morin, I. Stojmenovic and J. Urrutia, "Routing with guaranteed delivery in ad hoc wireless networks," in Proc. 3rd ACM Intl. Workshop on Discrete Algorithms and Methods for Mobile Computing and Communications DIAL M99, pp. 48-55, 1999.

[4] J. Bruck, J. Gao and A. Jiang, "Localization and routing in sensor networks by local angle information," manuscript, 2004.

[5] D. Dubhashi, O. Haggstrom and A. Panconesi, "Connectivity properties of Bluetooth wireless networks," manuscript, 2003.

[6] M. Franceschetti, Wireless Networks: from the Collective Behavior to the Physics of Propagation, Ph.D. dissertation, http://www.paradise.caltech.edu/papers/thesis007.pdf, 2002.

[7] J. Gao, L. J. Guibas, J. Hershberger, L. Zhang and A. Zhu, "Geometric spanner for routing in mobile networks," in Proc. 2nd ACM Symposium on Mobile Ad Hoc Networking and Computing (MobiHoc'01), pp. 45-55, 2001.

[8] E. N. Gilbert, "Random plane networks", J. SIAM, vol. 9, pp. 533-543, 1961.

[9] C. Gotsman and Y. Koren, "Distributed graph layout for sensor networks," in Proc. International Symposium on Grpah Drawing, Sept., 2004.

[10] G. Grimmett, Percolation, Springer-Verlag, Berlin Heidelberg, 1999.

[11] O. Haggstrom and R. Meester, "Nearest neighbor and hard sphere models in continuum percolation," Random Structures and Algorithms, vol. 9, no. 3, pp. 295-315, 1996.

[12] T.-C. Hou and V. O. K. Li, "Transmission range control in multihop packet radio networks," IEEE Trans. Commun., vol. COM-34, no. 1, pp. $38-44,1986$.

[13] B. Karp and H. Kung, "GPSR: greedy perimeter stateless routing for wireless networks," in Proc. Mobicom'00, Aug., 2000.

[14] Y. Ko and N. Vaidya, "Location-aided routing (LAR) in mobile ad hoc networks," in Proc. Mobicom'98, Oct., 1998.

[15] E. Kranakis, H. Singh and J. Urrutia, "Compass routing on geogmetric networks," in Proc. 11th Canadian Conf. Computational Geometry, Vancouver, Canada, Aug., 1999. 
[16] L. Li, J. Y. Halpern, P. Bahl, Y.-M. Wang and R. Wattenhofer, "Analysis of a cone-based distributed topology control algorithm for wireless multihop networks," in Proc. the Annual ACM Symp. Principles of Distributed Computing, pp. 264-273, New Port, Rhode Island, USA, Aug., 2001.

[17] A. Mann and J. Ruckert, "Transmission range control for packet radio networks or why magic numbers are distance dependent," Lecture Notes in Control and Information Sciences, vol. 143, pp. 818-830, 1990.

[18] M. Mauve, J. Widmer and H. Hartenstein, "A survey on position-based routing in mobile ad-hoc networks," IEEE Network, vol. 15, no. 6, pp. 30-39, 2001.

[19] R. Meester and R. Roy, Continuum Percolation, Cambridge University Press, 1996.

[20] C.-C. Shen, C. Srisathapornphat, R. Liu, Z. Huang, C. Jaikaeo and E. L. Lloyd, "CLTC: a cluster-based topology control framework for ad hoc networks," IEEE Trans. Mobile Computing, vol. 3, no. 1, pp. 18-32, 2004.

[21] H. Takagi and L. Kleinrock, "Optimal transmission ranges for randomly distributed packet radio terminals," IEEE Trans. Commun., vol. COM-32, no. 3, pp. 246-257, 1984.

[22] R. Wattenhofer, L. Li, P. Bahl and Y.-M. Wang, "Distributed topology control for power efficient operation in multihop wireless ad hoc networks," in Proc. IEEE Infocom 2001, pp. 1388-1397, Apr., 2001.

[23] F. Xue and P. R. Kumar, "The number of neighbors needed for connectivity of wireless networks," Wireless Networks, vol. 10, no. 2 , pp. 169-181, 2004.

[24] M. Zorzi and R. R. Rao, "Geographic random forwarding (GeRaF) for ad hoc and sensor networks: multihop performance," IEEE Trans. Mobile Computing, vol. 2, no. 4, pp. 337-348, 2003. 